\title{
Controle de infecção hospitalar: histórico e papel do estado ${ }^{1}$
}

\section{Control of hospital infection: description and paper of the state}

\author{
Control de la infección del hospital: descripción y papel del estado
}

\author{
Rosangela de Oliveira', Sônia Ayako Tao Maruyama"I
}

\section{RESUMO}

As Infecções Hospitalares - IH são complicações relacionadas à assistência à saúde e se constituem na principal causa de morbidade e mortalidade hospitalar, gerando prejuízos aos usuários, à comunidade e ao Estado. Nessa perspectiva, este artigo tem por objetivo abordar a história das instituições hospitalares e suas relações com as práticas de controle de infecção, além de identificar o papel do Estado, dos profissionais de saúde e dos usuários dos serviços no controle das $\mathrm{IH}$. Trata-se de um estudo de revisão de literatura com base nos artigos encontrados no Scielo, em textos publicados sobre Vigilância Sanitária e em bibliografia pertinente, utilizando os descritores "infecção hospitalar", "assistência hospitalar" e "vigilância sanitária". Do material levantado procedemos à leitura de forma a identificar aqueles que nos subsidiassem o entendimento da relação entre infecção hospitalar, as instituições hospitalares e o papel do Estado. Como resultado foi possível relacionar "Os hospitais e as infecções hospitalares" e "O controle da IH e o papel do Estado". As informações acessadas pelo estudo nos possibilitaram compreender que o controle das IH envolve um esforço conjunto de diferentes sujeitos e instâncias, ou seja, profissionais de saúde, gestores, Estado e comunidade.

Palavras chave: Infecção hospitalar; Vigilância sanitária; Assistência hospitalar.

\section{ABSTRACT}

The nosocomial infections are complications related to health assistance and they constitute the main cause of morbidity and nosocomial mortality, originating prejudices to the users, the community and the State. In this perspective, the purpose of this article has for objective to approach the history about hospital institutions and its relations with the infection control practices, beyond identify the paper of State, of the professionals of health and users of the service in its control. One is about a study of literature revision on the basis of articles found in the Scielo, texts published on Sanitary Monitoring and pertinent bibliography, using the describers "hospital infection", "hospital assistance" and "sanitary monitoring". From the obtained material we proceed the reading to identify those that subsidized us in the comprehension of the relation among nosocomial infection, the hospital institutions and the State's paper. As result, it was possible to relate "The hospitals and nosocomial infections" and "The control of the nosocomial infections and the State's paper". The information had access for the study allowed us to understand that the control of the $\mathrm{IH}$ involves a joint effort of different citizens and instances, that is, professionals of health, managers, State and community.

Key words: Nosocomial infections; Sanitary vigilance; Nosocomial welfare work.

\section{RESUMEN}

Las infecciones del hospital son complicaciones relacionadas con la asistencia a la salud y si constituyen en la causa principal de la morbidad y mortalidad del hospital, gerando perjuicio a los usuarios, a la comunidad y al Estado. En esta perspectiva, el propósito del artículo tiene el objetivo acercarse a la historia de las instituciones nosocomiales y su relaciones con las practicas de control de las infecciones, más allá de identificar el papel del Estado, los profesionales de la salud y los usuarios de los servicios en su control. Se trata de un estudio de la revisión de la literatura en base de los artículos encontrados en Scielo, textos

\footnotetext{
Trabalho vinculado ao Grupo de Pesquisa Enfermagem Saúde e Cidadania - GPESC (Integralidade, Práticas de Atenção e de Gestão e Evento-sentinela), financiado pelo CNPq

' Enfermeira. Mestranda do Programa de Pós-Graduação da Faculdade de Enfermagem da Universidade Federal de Mato Grosso. Cuiabá - MT. E-mail: oliveirapedroso@pop.com.br. " Enfermeira. Doutora em Enfermagem Fundamental. Professora da Faculdade de Enfermagem da Universidade Federal de Mato Grosso. Cuiabá - MT. E-mail: soniayako@uol.com.br.
} 
publicados en la supervisión sanitaria y la bibliografía pertinente, usando los descriptores "infección del hospital", "asistencia del hospital" y "la vigilancia sanitaria". Del material levantado procedemos la lectura para identificar los que nos subvencionaron en la comprensión de la relación entre la infección nosocomial, las instituciones del hospital y el papel de Estado. Como resultado, fue posible relacionar "Los hospitales y las infecciones nosocomiales" y "El

\section{NTRODUÇÃO}

A assistência à saúde vem, ao longo dos tempos, evoluindo com os avanços científicos e tecnológicos, e tem refletido em melhoria das ações de saúde para a população. Porém, se por um lado se observa o desenvolvimento científico-tecnológico nas ações de saúde, por outro, tem-se observado que problemas antigos ainda persistem como é o caso das infecções hospitalares $^{(1)}$.

A infecção hospitalar é definida pela Portaria MS $n^{\circ} 2616$ de 12/05/1998 como "aquela adquirida após a admissão do paciente e que se manifeste durante a internação ou após a alta, quando puder ser relacionada com a internação ou procedimentos hospitalares". Elas representam complicações relacionadas à assistência à saúde e constituem a principal causa de morbidade e mortalidade hospitalar, aumentando o tempo de internação dos pacientes e, com isso, elevam os custos dos hospitais e reduzem a rotatividade de seus leitos $^{(2)}$. Os procedimentos cada vez mais invasivos, ao uso indiscriminado e a resistência aos antimicrobianos são fatores que apontam as infecções hospitalares como um grave problema de saúde pública ${ }^{(3-4)}$.

No Brasil, sua prevalência exata de um modo geral ainda é desconhecida ${ }^{(4)}$, entretanto, um inquérito nacional realizado pelo Ministério da Saúde revelou que entre as instituições avaliadas, a taxa de infecção hospitalar variou de $13 \%$ a $15 \%{ }^{(5-6)}$. Esta se apresenta bastante alta se compararmos a um estudo levantado pela Organização Mundial de Saúde (OMS) que encontrou em 14 países, entre 1983-1985, taxa média de $8,7 \%{ }^{(7)}$, ou seja, o estudo realizado no Brasil revelou que este apresenta praticamente o dobro de casos de infecção hospitalar em relação aos outros países control de las infecciones nosocomiales y del papel de estado". Las informaciones accesadas por el estudio permitieron comprender que el control del IH implica un esfuerzo conjunto de diversos ciudadanos y de los sujetos, es decir, profesionales de la salud, encargados, estado y comunidad.

Palabras clave: Infección hospitalaria; Vigilancia sanitaria; Atención hospitalaria.

estudados, demonstrando a necessidade de medidas mais eficazes para a redução dessas taxas no país.

A infecção hospitalar há muito tempo tem sido motivo de preocupação entre os órgãos governamentais e, embora a sua regulamentação tenha ocorrido na década de 80 , a problemática no país continua ainda sendo negligenciada ${ }^{(2,8,9)}$.

Neste contexto, com base nas práticas vivenciadas como profissional de saúde em diferentes instituições hospitalares, observamos aspectos do cotidiano dos profissionais de saúde e dos usuários que se relacionam com a problemática das infecções hospitalares. Diante da realidade apresentada e das reflexões, surgiram questões como: como as infecções hospitalares surgiram? Quais as responsabilidades do poder público no controle da infecção hospitalar? Quais mecanismos de regulação e controle de serviços e produtos 0 poder público se utiliza?

O que nos motivou a realizar o estudo foi a necessidade de compreender as infecções hospitalares a partir da sua constituição e do papel do Estado no seu controle, aprofundando os conhecimentos relativos a esta temática que faz parte da nossa atividade profissional, no intuito de buscar um melhor entendimento para esse importante problema de saúde pública que implica na garantia da qualidade e da segurança na prestação de serviços de saúde.

Assim, buscamos atender a dois objetivos: abordar a história das instituições hospitalares e suas relações com as práticas de controle de infecção, além de identificar o papel do Estado, dos profissionais de saúde e dos usuários dos serviços no controle das IH.

Pretendemos com este artigo subsidiar reflexões acerca das práticas de controle de 
infecção hospitalar bem como ressaltar a importância de co-responsabilizar as pessoas envolvidas no contexto destas práticas, ou seja, gestores, profissionais de saúde, usuários e familiares, enfim a sociedade, de forma a qualificar a atenção nas instituições que prestam assistência a saúde.

\section{METODOLOGI A}

Trata-se de um estudo de revisão de literatura, com base em artigos encontrados no Scielo e, em textos publicados nos Manuais da Vigilância Sanitária e em bibliografia pertinente, utilizando os descritores "infecção hospitalar", "assistência hospitalar" e "vigilância sanitária". Do material levantado selecionamos aqueles que se enquadravam no período de 1997 a 2006. Após a leitura dos mesmos, selecionamos 19 textos que abordavam a temática em questão. Para análise e síntese seguimos os seguintes passos ${ }^{(10)}$ : leitura exploratória para reconhecer do que se tratava o texto; em seguida procedemos a uma leitura seletiva, selecionando o material em busca de informações que pudessem estar relacionados aos objetivos e a temática proposta. Após a leitura geral dos textos, procedemos ao fichamento segundo os objetivos propostos, ou seja, que nos subsidiassem o entendimento da relação entre infecção e as instituições hospitalares e o papel do Estado. Seguimos para uma leitura analítica que nos possibilitasse a construção de categorias e, posteriormente, realizamos uma leitura interpretativa, ou seja, procuramos dar significado aos dados encontrados. Como resultado foi possível relacionar dois aspectos: "Os hospitais e as infecções hospitalares" e "O controle da IH e o papel do Estado"(10).

\section{OS HOSPITAIS E AS INFECÇÕES HOSPITALARES}

O aparecimento das infecções hospitalares é tão antigo quanto o surgimento dos hospitais. Data aproximadamente do ano 330 a.C. no Império Romano, a existência do primeiro hospital urbano, embora tenha sido relatada a construção do primeiro hospital em 394 a.C. na periferia de Roma Posteriormente, muitos outros foram criados, como o Hotel-Dieu na França, que possuía cerca de 1.200 leitos, muitos dos quais eram compartilhados com outros doentes ${ }^{(6)}$. Ressaltamos a utilização do termo "hospital" por conveniência didática, haja vista que tal denominação não se aplicava a esta instituição nos primórdios de sua existência.

Conforme o Concílio de Nicéia, os hospitais eram construídos próximos às catedrais ${ }^{(11)}$, caracterizando uma função caritativa, de assistência aos pobres, inválidos, peregrinos e doentes. Esses espaços de segregação e de exclusão, similares a albergues ou asilos, eram considerados fonte inesgotável de doença devido às características sanitárias e de assistência precárias, não se fazendo presentes, portanto, nem o médico e nem a atividade terapêutica ${ }^{(12)}$.

Não dispunham de nenhuma forma de sistematização assistencial que evitasse os contágios entre as pessoas ali assistidas, favorecendo a disseminação de doenças, especialmente as de caráter infeccioso ${ }^{(12)}$. O hospital era mais do que local de cura e cuidado, uma fonte de doença e local de morte.

Desde o período a.C., na Roma Antiga e na Idade Média, a assistência nos hospitais era prestada quase sempre por mulheres, como religiosas, prostitutas e outras pessoas sem qualquer tipo de qualificação e remuneração. Para aquelas consideradas pecadoras, servia como um meio para a remissão dos seus pecados e merecimento de indulgências ${ }^{(13)}$.

Por terem caráter social, os hospitais não eram utilizados pelas famílias mais abastadas. Estas, realizavam os cuidados e a assistência aos seus enfermos no próprio domicílio. Até meados do Século XVIII, o hospital não era um local para o doente se curar e sim um local para assistência aos pobres que estavam morrendo ${ }^{(12)}$.

Paralelamente às condições precárias pelas quais se conformavam os hospitais até meados do século XVIII, se observou a ocorrência das infecções hospitalares, ou seja, as pessoas doentes assistidas nos hospitais, passavam a desenvolver outras doenças em função da hospitalização.

A infecção surge nos hospitais como uma conseqüência das precárias condições em que as pessoas eram dispostas e atendidas naqueles ambientes. A precariedade das condições por 
sua vez, contribuiu para a evolução do conhecimento sobre o hospital e sua finalidade, que, gradativamente, passa a ter uma nova função na assistência à saúde.

A mudança desse paradigma caritativoassistencial ocorre no final do Séc. XVIII, com a conscientização de que o hospital poderia e deveria ser um instrumento de terapêutica, não pela ação sobre o doente ou a doença, mas pelos efeitos negativos que ele causava, como as desordens econômico-sociais ${ }^{(12)}$.

A partir de inquéritos a pedido da Academia de Ciências em outros hospitais da Europa, inicia-se a reforma e reconstrução do Hotel-Dieu de Paris. Surgem também novas concepções quanto à relação entre fenômenos patológicos e espaciais, como segregação de doentes de acordo com a nosologia, cuidados com contaminações e o ambiente, de forma a evitar os fatos patológicos próprios dos hospitais $^{(12)}$.

A emergência do capitalismo corrobora para o processo da reestruturação dos hospitais, no objetivo de valorizar o corpo como objeto principal para as forças de trabalho necessárias ao modelo capitalista $^{(8)}$ e reduzir custos das instituições hospitalares que mantinham ociosos e necessitados, encarecendo a sua manutenção(12).

Os hospitais iniciam sua gradual reestruturação, de um local de caridade para um local de cura, de observação, de saberes e de disciplina, com a inserção dos médicos, melhorando as condições de atendimento, tornando-os instituição mais funcional, internando doentes somente com indicação médica para evitar a superlotação e contribuindo para o ensino. Essa disciplinarização instituída com a entrada dos médicos nos hospitais, constitui com o saber médico, o início das relações de poder e da hegemonia médica, que se estabelece progressivamente na hierarquização da classe.

Começam a surgir as primeiras medidas básicas de controle de infecção para o atendimento ao novo propósito do hospital. No contexto histórico das infecções hospitalares, em 1847, grande importância representou I gnaz Philipp Semmelweis (1818-1865), médico cirurgião húngaro, que em Viena, em meados do Séc. XIX, observando as altas taxas de infecção puerperal em mulheres que haviam sido tratadas por médicos que antes haviam realizado necropsias, instituiu a rotina de higiene de mãos com solução clorada. Neste simples ato, conseguiu reduzir as taxas de infecção de $11,4 \%$ para $1,3 \%$ em um período de sete meses ${ }^{(6)}$. Ressalte-se que, em 1843, Oliver Wendel Holmes fez esta mesma relação que Semmelweis, embora convincente e com argumentos lógicos, foi tratado com indiferença e hostilidade pela classe médica, não conseguindo êxito na época ${ }^{(5,11)}$.

A partir da contribuição do trabalho de Semmelweis (1860), reforçada por Lister (1867) e seguidamente por outros pesquisadores, foi-se estabelecendo a relação que havia entre os pacientes internados que apresentavam as infecções e os óbitos ${ }^{(14)}$.

Na Inglaterra, no final do Séc. XIX, Florence Nightingale representou significativa importância histórica com sua contribuição na (re)organização dos hospitais e, conseqüentemente, na implantação de medidas para o controle das infecções hospitalares, como a preocupação voltada para os cuidados de higienização, o isolamento dos enfermos, o atendimento individual, a utilização controlada da dieta e a redução de leitos no mesmo ambiente, instituindo medidas de organização, sistematização do atendimento e treinamento de pessoal, especialmente as práticas higiênicosanitárias que estabeleceu e que colaboraram para a redução das taxas de mortalidade hospitalar da época ${ }^{(8,13)}$. Considerada a precursora da enfermagem moderna, era dotada de um talento raro, muito à frente das pessoas de sua época, e seus conhecimentos e vivências práticas na assistência à saúde tem contribuído até hoje, várias décadas após a sua morte.

Especificamente no Brasil, as primeiras referências ao controle da contaminação hospitalar, termo utilizado na época, surgiram na década de 50, aproximadamente em 1956, com questionamentos quanto a medidas ambientais, práticas relativas aos procedimentos invasivos como as técnicas assépticas, processos de esterilização de material hospitalar e o aparecimento de microorganismos resistentes pelo uso indiscriminado de antibióticos ${ }^{(5-6)}$. Nas escolas 
médicas, esta temática era pouco abordada, fato que persiste ainda nas instituições formadoras de profissionais de saúde ${ }^{(6,15)}$.

Nas últimas décadas do Séc. XIX no Brasil, os índices de infecção verificados entre os pacientes submetidos a procedimentos cirúrgicos chegavam a até $90 \%$ e suas causas se relacionavam a falta de regras básicas de higiene e de isolamento dos doentes portadores de doenças infecciosas, causando o que se denominavam na época de "gangrenas dos hospitais", representando a principal causa de mortalidade nos hospitais brasileiros ${ }^{(6)}$.

Os primeiros relatos no país quanto à ocorrência de infecção hospitalar, surgiram na década de 50, e, embora se utilizasse o termo "contaminação hospitalar", referiam como causas a esterilização do material hospitalar, o uso indiscriminado de antibióticos e o surgimento de microorganismos resistentes ${ }^{(5-6)}$.

A partir de 1968 surgem as primeiras Comissões de Controle de Infecção Hospitalar ( $\mathrm{CClH})$ no país, vinculadas a instituições de ensino inicialmente. Em 1976, o governo determina a necessidade de criação de $\mathrm{CClH}$ nos hospitais próprios da previdência, mas a medida não causa impacto pela falta de fiscalização.

A década de 80 caracteriza-se por um grande avanço no controle de infecção, ocorrendo vários eventos relativos ao tema, levando a criação de várias $\mathrm{CCIH}$ nos hospitais brasileiros $^{(5-6)}$.

Em 1983, o Ministério da Saúde, pressionado pelos fatos veiculados na imprensa relativos a casos de infecções hospitalares, emitiu a Portaria MS no 196/1986 que recomendava aos hospitais brasileiros a criação de $\mathrm{CCIH}$.

Em 1985, a morte do recém-eleito Presidente da República, Tancredo Neves, por septicemia devido a uma infecção pós-cirúrgica, causou grande repercussão nacional, corroborando para que o Ministério da Saúde implementasse ações e projetos que mudassem o panorama e os rumos do controle de infecção no país. Desencadearam-se ações como o levantamento das instituições brasileiras que já tinham $\mathrm{CClH}$ operacionalizadas, capacitação de multiplicadores, intercâmbio de conhecimentos entre os profissionais de saúde, elaboração de manuais e normas técnicas ${ }^{(6)}$. Em 1989 ocorreu - I Congresso Brasileiro sobre Infecção Hospitalar em São Paulo, como conseqüência do desenvolvimento desse conhecimento entre os profissionais e na constituição de um novo mercado de trabalho que se criava ${ }^{(5)}$.

Os anos 90 marcaram um progressivo desgaste no Programa de Controle de Infecção Hospitalar Brasileiro, mesmo com a publicação da Portaria MS no 930/1992. A política de descentralização das ações de saúde, amparada pela Lei no 8.080/1990, provocou a fragmentação e dispersão das bases de apoio em controle de infecção hospitalar do Ministério da Saúde ${ }^{(6)}$.

O efeito dessa descentralização culminou na formação de núcleos de profissionais em alguns Estados com o intento de manter trocas de experiências, dando origem a várias associações de profissionais em controle de infecção ${ }^{(6)}$.

Em conseqüência do não cumprimento da Portaria MS no 930/1992 por grande parte dos hospitais brasileiros, o Ministério da Saúde emitiu a Lei Federal no 9.431/1997 que determinava a obrigatoriedade de manutenção de Programas de Controle de Infecção Hospitalar em todos os hospitais do país, mas vetava a obrigatoriedade de serviços de controle de infecção e busca ativa de casos.

Quase dez anos após a emissão da Lei Federal no 9.431, o Ministério da Saúde emite a Portaria no. 2.616/1998, ainda em vigor, que mantém a obrigatoriedade da existência de um $\mathrm{PClH}$ em todos os hospitais do país, trata da organização e competências da Comissão de Controle de Infecção Hospitalar - $\mathrm{CClH}$ e do Programa de Controle de Infecção Hospitalar $\mathrm{PClH}$, estabelece os conceitos e critérios diagnósticos das Infecções Hospitalares, dá orientações sobre a vigilância epidemiológica das infecções hospitalares e seus indicadores, faz recomendações sobre a higiene das mãos e enfatiza a observância de publicações anteriores do Ministério da Saúde quanto ao uso de germicidas, microbiologia, lavanderia e farmácia.

Com a necessidade de centralizar ações de regulação de alimentos e medicamentos inicialmente e, posteriormente, de produtos e serviços de interesse da saúde, em 1999 foi 
criada a Agência Nacional de Vigilância Sanitária (ANVISA), autarquia ligada ao Ministério da Saúde, em cujas atribuições inclui também o controle de infecção hospitalar em nível federal, com suporte às Secretarias Estaduais por meio de apoio técnico, capacitações, expedição de normas e legislações, consolidação de informações e promoção da socialização das informações pertinentes.Para a instrumentalização mais efetiva das ações de fiscalização sanitária no monitoramento das ações de Controle das Infecções nos estabelecimentos hospitalares, a ANVISA emitiu a Resolução RDC no. 48/2000, para estabelecer a sistemática para avaliação/inspeção dos Programas de Controle de Infecção Hospitalar. Serviu para dar suporte à fiscalização sanitária e aos profissionais das $\mathrm{CClH}$ dos hospitais.

A deficiência de indicadores de infecções hospitalares $^{(16)}$ levou a ANVISA a desenvolver o Sistema de Informações para o Controle de Infecção em Serviços de Saúde (SINAIS), cujo objetivo é conhecer o perfil epidemiológico e as taxas de infecções hospitalares nos hospitais, buscando uniformizar e padronizar os indicadores com possibilidade de acompanhamento, além de servir como instrumento de orientação para implantação das ações que visam diminuir sua incidência e gravidade nos serviços de saúde, medir sua eficácia e monitorar a qualidade da assistência hospitalar e riscos.

$\mathrm{Na}$ prática, mesmo com a existência de legislações e normativas para o exercício do controle sanitário, verificamos a interferência de vários discursos influindo nas práticas, sejam de ordem política, econômica, jurídica ou técnica, acarretando confrontos dos mais variados, que acabam por interferir nas ações da regulação sanitária. Esses discursos, embora não possuam a capacidade de perpassar domínios técnicos e científicos, constituem relações de forças capazes de suprimir a execução do exercício institucional efetivo de controle de infecção hospitalar no Brasil.

\section{O CONTROLE DE I NFECÇÃO HOSPITALAR E O PAPEL DO ESTADO}

O sistema de fiscalização sanitária, originou-se na Europa entre os séculos XVII e XVIII, na época denominada "polícia sanitária", pela necessidade de manter a paz, o crescimento ordenado, o controle das riquezas e as condições de manutenção da saúde que surgem em função do crescimento das cidades $^{(12)}$.

Dotada de um conjunto de regulamentos e de instituições múltiplas, na área da saúde, visava a regulamentação do exercício da profissão médica, também realizado por curiosos sem formação específica ${ }^{(13)}$, combatendo o charlatanismo, promovendo o saneamento das cidades, fiscalizando as embarcações, os cemitérios e a comercialização de alimentos e evitando que as doenças se propagassem entre a população(17).

No Brasil, a preocupação sanitária data entre os séculos XVIII e XIX, pouco após o seu surgimento na Europa, embora já no século XVI tenham ocorrido ações de fiscalização e de punição de curta efetividade ${ }^{(17)}$.

Para o controle sanitário de serviços e produtos de interesse e de atenção à saúde, o poder público tem como responsabilidade fazer com que as normas e legislações relacionadas sejam aplicadas para evitar a exposição da população a riscos. O descumprimento do exercício atribuído ao agente público, implica na desconsideração e responsabilidade que the foi imputada, constituindo obrigação legal do Estado promover a indenização dos prejuízos causados em razão da não execução das atividades públicas atribuídas ${ }^{(18)}$.

As ações de Vigilância Sanitária englobam a fiscalização e/ou vistoria, o licenciamento, a imposição de penalidades (notificação, apreensão, infração e interdição), o trabalho educativo (educação em saúde), coleta, processamento e divulgação das informações de interesse para a Vigilância Sanitária e Epidemiológica, dentre outros, observados os ditames legais e normativos das três instâncias (federal, estadual e municipal) que embasam seus mecanismos.

As práticas de fiscalização sanitária, inicialmente, conformavam-se de forma essencialmente policialesca, caracterizando o "exercício de polícia". Atualmente, a forma de atuação vem sendo modificada, numa concepção de trabalho voltada para a educação sanitária da população, com o objetivo de tornar mais efetiva e eficaz a atuação da Vigilância 
Sanitária, socializando o corpo social quanto à noção das possibilidades dos riscos a que estão expostos nas diferentes práticas sob controle sanitário e de suas intervenções quando necessárias ${ }^{(19)}$. A participação e o controle popular como constituição de uma relação de poder mútua se imbrica com as ações de controle do órgão fiscalizador, garantindo sua participação ativa no processo como representante dos interesses da sociedade como consumidores e, conseqüentemente, colaborando na otimização e resolutividade das práticas fiscais.

A atuação do órgão de Vigilância Sanitária representa o cumprimento do papel que cabe ao Estado que é o monitoramento de controle de ambientes, produtos e serviços de interesse à saúde, embasados em legislações e normativas das três instâncias.

Os hospitais são serviços complexos e envolvem um grande número de legislações e normativas, devido à sua especificidade complexa e característica. Muitas dessas legislações e normativas são criadas para setores ou serviços específicos, dentro da própria instituição. Devido à extensão de atividades realizadas na fiscalização, procuraremos destacar os pontos principais da inspeção sanitária nesses serviços.

O principal aspecto verificado diz respeito às questões higiênicas do estabelecimento, requisito considerado básico e fundamental para o funcionamento do serviço, pois sua realização efetiva e adequada permeia todos os outros processos que ocorrem no hospital. Embora seja essa uma atividade básica, ainda representa um grande problema em muitos serviços, constituindo um importante foco de disseminação de partículas e microorganismos, favorecendo a ocorrência das infecções hospitalares.

Todos os demais processos e procedimentos realizados no hospital, tanto os que envolvem a assistência aos clientes como os demais, são criteriosamente avaliados pela importância no contexto assistencial.

Quanto à estrutura física, é de praxe a aprovação prévia do projeto arquitetônico do estabelecimento pelo órgão sanitário quando em situações de construção, reforma, adequações ou ampliações, permitindo sua construção dentro das normas sanitárias requeridas na RDC/ANVISA no 50 de 21/02/2002. Em casos cujas construções são antigas e fora dos padrões sanitários vigentes, após análise prévia do projeto, são sugeridas adequações possíveis de serem realizadas para que estejam o mais próximo possível do estabelecido nas normas. Tais análises permitem buscar estratégias de forma a permitir a minimização de riscos de infecções hospitalares, especialmente em setores considerados críticos como as terapias intensivas e os centros cirúrgicos por exemplo, uma vez que são nesses setores que os pacientes são submetidos a procedimentos invasivos, estando conseqüentemente, mais expostos às infecções hospitalares. Nessa perspectiva, é importante a participação da $\mathrm{CClH}$ do estabelecimento nas discussões dos projetos a fim de contribuir com seus conhecimentos técnicos específicos.

Quando evidenciamos problemas decorrentes da estrutura física, seja no que se refere a fluxos, espaços ou acabamentos utilizados, o serviço é notificado a encaminhar projeto para avaliação, para que, em seguida, se procedam às adequações necessárias.

A inexistência de normalização e socialização das diferentes práticas são comumente verificadas e implicam na ineficiência do serviço de instituir as normas e rotinas (Procedimentos Operacionais Padrão POP) para o seu funcionamento, que devem ser seguidas uniformemente por todos os profissionais. Sua elaboração pode ser feita em parceria com o Serviço de Educação Continuada e a Comissão de Controle de Infecção Hospitalar, seguido de capacitação para a socialização das informações instituídas no serviço aos profissionais de saúde ${ }^{(2,15)}$.

A inatividade ou ineficiência da Comissão de Controle de Infecção Hospitalar $(\mathrm{CCIH})$ é outro fato muito comum encontrado nos hospitais e que acaba acarretando prejuízos à instituição, seja pela falta de capacitação ou perfil dos profissionais para atuação na área ou por falta de conscientização do gestor quanto às necessidades propostas pela $\mathrm{CCl}$, nem sempre atendidas. Muitas vezes ocorre da $\mathrm{CClH}$ não dispor de um Programa de Controle de Infecção Hospitalar ( $\mathrm{PCl} H)$ implantado e implementado, 
trabalhando sem um direcionamento para suas ações.

Muitos hospitais ainda não atentaram para a importância da contribuição da $\mathrm{CClH}$ na assessoria administrativa da instituição, diagnosticando e vigiando a freqüência e distribuição das infecções hospitalares entre os pacientes internados e egressos, intervindo através da implantação e/ou implementação de medidas de controle de infecção, visando garantir a qualidade e segurança da sua assistência(16).

As práticas sanitárias evidenciam a necessidade de implementação de medidas de maior impacto no funcionamento dos serviços hospitalares, com um enfoque maior para a institucionalização de práticas de controle de infecção, que, muitas vezes, não tem o respaldo administrativo para a sua devida operacionalização. Há necessidade contínua de revisões e implementação das práticas de prevenção e controle, em virtude da utilização de procedimentos cada vez mais sofisticados e invasivos, da virulência dos microorganismos, do uso inadvertido de antimicrobianos e da resistência microbiana ${ }^{(2)}$.

Além das práticas relacionadas aos procedimentos técnicos, é de fundamental importância para o êxito das ações de controle de infecção, o envolvimento, a participação e a integração da $\mathrm{CCIH}$ com os serviços de apoio do hospital ${ }^{(20)}$, como, especialmente, o laboratório de microbiologia, a farmácia hospitalar, a lavanderia, a nutrição e o serviço de limpeza, uma vez que estão também envolvidos com o controle de infecção. Nessa perspectiva, relacionamos alguns Programas básicos a serem instituídos nos hospitais, como Programa de Controle Médico e Saúde Ocupacional (PCMSO), Programa de Prevenção de Riscos Ambientais (PPRA), Programa de Gerenciamento de Resíduos de Serviços de Saúde (PGRSS), Controle Integrado de Pragas e Vetores, Programa de Manutenção Preventiva e Corretiva de Equipamentos Médico-hospitalares e Programa de Manutenção e Controle de Limpeza dos Condicionadores de $\mathrm{Ar}$ ou Sistemas de Ventilação, em especial nos setores críticos.

Assim, o Estado busca através de diversos mecanismos regulatórios, fiscalizar e normatizar as práticas de controle de infecção hospitalar, visando garantir à população o acesso a serviços de saúde que disponibilizem atendimento seguro e de qualidade.

\section{CONSI DERAÇÕES FINAIS}

A infecção hospitalar é um grave problema de saúde pública e representa um grande desafio a ser enfrentado pelo poder público para a execução das ações de prevenção e controle de infecção nas instituições hospitalares.

A realidade de muitos hospitais ainda é deficiente sob aspectos relativos às questões sanitárias legais e normativas, e principalmente, quando se trata da inexistência de Comissões e de Programas de Controle de Infecção Hospitalar para a aplicação das medidas de prevenção e controle desses eventos.

Defendemos a importância dos fatores "vontade e iniciativa" dos sujeitos, entretanto, não desvinculamos a valorização das ações de capacitação e conscientização dos agentes (trabalhadores e usuários) articulados em consonância com os gestores dos serviços.

Nesse sentido, pactuamos da necessidade de institucionalização dessa temática nas unidades de formação de profissionais de saúde no Brasil, permitindo que os profissionais de saúde possam atuar com maior respaldo científico no assunto. Sabemos também que essa atitude implica em decisões políticas que devem ser instigadas num esforço coletivo entre profissionais de saúde e população em benefício do bem comum.

Assim, a problemática das infecções hospitalares requer também mudanças de ordem governamental, como a criação de uma política para o controle de infecção de maior efetividade, que vá além do estabelecimento de mecanismos legais e normativos para a sua regulação, que envolva a população usuária dos serviços, tornando-a partícipe no processo.

É necessário refletir sobre todas as estratégicas possíveis que possam contribuir para a mudança do atual panorama que se apresenta, como: a inserção da temática nos currículos dos cursos de graduação na área de saúde; a ampliação dos investimentos na disponibilização de cursos de pós-graduação em controle de infecção para profissionais de saúde, especialmente fora dos grandes centros; garantias legais no reconhecimento e autonomia 
do profissional controlador de infecção; revisão da legislação vigente objetivando o cumprimento efetivo das medidas de prevenção e controle, tanto para estabelecimentos públicos como privados; investimentos em pesquisas, seminários e atualizações.

Acreditamos ainda que não baste investimentos em altas tecnologias em saúde sem considerar a importância de se investir no potencial humano como um elemento fundamental para a o desenvolvimento de práticas de controle de infecção que culminem numa assistência segura e qualificada, que minimiza a permanência na internação, o aumento dos custos hospitalares, o sofrimento dos pacientes e de suas famílias, trazendo repercussões para toda a sociedade.

\section{REFERÊNCI AS}

1. Andrade D, Angerami ELS, Padovani CR. Condição microbiológica dos leitos hospitalares antes e depois de sua limpeza. Rev. Saúde Pública. 2000; 34(2): 163-9.

2. Andrade D, Angerami ELS. Reflexões acerca das Infecções Hospitalares às portas do Terceiro Milênio. Medicina. 1999; 32:492-7.

3. Starling CEF, Fialho AS, Alves Junior AA, Moura JA, Couto BRGM. Impacto das Infecções Hospitalares na Lucratividade de Hospitais Privados Brasileiros. Prática Hospitalar. 2004;6(34): 77-80.

4. Turrini RNT, Santo AH. Infecção Hospitalar e causas múltiplas de morte. J. Pediatr. (Rio J.). 2002; 78(6): 485-90

5. Rodrigues EAC. Histórico das Infecções Hospitalares. In: Rodrigues EAC. Infecções Hospitalares: Prevenção e Controle. São Paulo: Sarvier; 1997. p. 3-27

6. Fernandes AT. Infecção Hospitalar e suas Interfaces na Área da Saúde. 1a edição. São Paulo: Atheneu; 2000.

7. Organização Pan-Americana da Saúde. Infecção Hospitalar. [Internet]. 2000 [cited 2006 jul 21] Available from: http://www. opas.org. br/sistema/fotos/hospitala1.PDF 8. Lacerda RA, Egry EY. As infecções hospitalares e sua relação com 0 desenvolvimento da assistência hospitalar: reflexões para análise de suas práticas atuais de controle. Rev. Latino-Am. Enfermagem. 1997;5(4): 13-23.
9. Mendonça AP, Fernandes MAC, Azevedo JMR, Silveira WCR, Souza ACS. Higiene das mãos: adesão dos profissionais de saúde em uma unidade de terapia intensiva neonatal. Acta Sci. Health Sci. 2003; 25(2): 147-53.

10. Gil AC. Como elaborar projetos de pesquisa. São Paulo: Atlas; 1991.

11. Couto RC, Pedroso ERP, Pedrosa TMG. História do Controle da Infecção Hospitalar no Brasil e no Mundo. In: Couto RC, Pedrosa TMG, Nogueira J M. Infecção Hospitalar e outras Complicações Não-infecciosas da Doença. 3a edição. Rio de Janeiro: Medsi; 2003. p. 3-8

12. Foucault M. Microfísica do poder. 10a edição. São Paulo: Graal; 1999.

13. Melo CMM. Divisão social do trabalho e enfermagem: A divisão social do trabalho na enfermagem. São Paulo: Cortez; 1986.

14. Couto RC, Pedrosa TMG. Epidemiologia Hospitalar. In: Couto RC, Pedrosa TMG, Nogueira JM. Infecção Hospitalar e outras Complicações Não-infecciosas da Doença. 3a edição. Rio de Janeiro: Medsi; 2003. p. 93-155

15. Pereira MS, Souza ACS, Tipple AFV, Prado MAA. Infecção Hospitalar e suas implicações para o cuidar da enfermagem. Texto contexto enferm. 2005; 14(2): 250-7.

16. Prade SS, Vasconcellos MM. Identificando fontes de dados nos Sistemas de Informações do SUS para a Vigilância Epidemiológica das IH. Informe Epidemiológico do SUS. 2001; 10(3): 121-128.

17. Costa EA, Rozenfeld S. Constituição da Vigilância Sanitária no Brasil. In: Rozenfeld S. (org.). Fundamentos da Vigilância Sanitária. Rio de Janeiro: Fiocruz; 2000. p. 15-40

18. Ferreira P. Responsabilidade Civil do Estado face às ações de Vigilância Sanitária em Serviços de Saúde. [especialização]. [Brasília]: ENSP; 2003. 60p.

19. Silva ZP, Coroa ML. Vigilância Sanitária: um histórico discursivo. REVISA. 2005; 1(1): 3-15. 20. Pereira MS. Avaliação de serviços de apoio na perspectiva do controle de infecção hospitalar. Rev. Eletr. Enf. [Internet]. 1999 [cited 2006 jul 21]; 1(1). Available from http://www.fen.ufg.br/revista/revista1_1/03.htm.

Artigo recebido em 05.02.07

Aprovado para publicação em 30.09.08 